\title{
Clinical implications for pro-GRP in small cell lung cancer. A single center experience
} journals.sagepub.com/home/jbm

\author{
Stefano Cavalieri ${ }^{1}$, Daniele Morelli ${ }^{2}$, Antonia Martinetti ${ }^{1}$, Giulia Galli ${ }^{1}$, Federico Nichetti ${ }^{1}$, Filippo de Braud ${ }^{1,3}$, Marco Platania $^{1}$ \\ ${ }^{1}$ Medical Oncology Department - National Cancer Institute of Milan - Fondazione IRCCS Istituto Nazionale Tumori, Milan - Italy \\ ${ }^{2}$ Laboratory Department - National Cancer Institute of Milan - Fondazione IRCCS Istituto Nazionale Tumori, Milan - Italy \\ ${ }^{3}$ Medical Oncology Department, University of Milan, Milan - Italy
}

\begin{abstract}
Background: Recently, pro-gastrin-releasing peptide (pro-GRP) became available as an alternative sensitive, specific and reliable tumor marker for patients with small cell lung cancer (SCLC), both in limited (LD) and diffuse disease (DD).

Methods: We retrospectively analyzed pro-GRP, neuron-specific enolase (NSE) and CEA in patients with SCLC and non-small cell lung cancer (NSCLC). Serum pro-GRP level was measured with electrochemiluminescence at our laboratory (cutoff $77.8 \mathrm{pg} / \mathrm{mL}$ ). Continuous variables were analyzed with the Mann-Whitney test, contingency data with Fisher's exact test. Receiver operator characteristic (ROC) curve analysis was performed to identify threshold values to set the highest sensitivity $(\mathrm{Sn})$ and specificity $(\mathrm{Sp})$ values.

Results: A total of 65 patients were studied (49 men, median age 67 years, range 27-79). Thirty-seven patients had SCLC (29 DD, 8 LD) and 28 advanced NSCLC. Median pro-GRP level was $919 \mathrm{pg} / \mathrm{mL}$ (range 22-147,350) in SCLC and $32 \mathrm{pg} / \mathrm{mL}$ (range 10-119.2) in NSCLC ( $p<0.0001)$. NSE was 4.38-fold higher in SCLC patients $(p=0.0005)$; CEA did not reveal significant differences between groups. Pro-GRP Sn and Sp were $86.4 \%$ and $96.4 \%$, respectively. With ROC curve analysis, a cutoff value of $329.3 \mathrm{pg} / \mathrm{mL}$ showed a $\mathrm{Sn}$ of $75.8 \%$ and $\mathrm{Sp}$ of $87.5 \%$ in discriminating DD from LD. Pro-GRP was not influenced by either liver metastases or renal impairment.

Conclusions: Pro-GRP is sensitive for SCLC diagnosis. Since high marker levels are related to high disease burden, pro-GRP may have a negative prognostic significance. Follow-up studies are required to define its role in clinical practice in monitoring responses to treatment and early relapses.
\end{abstract}

Keywords: Marker, Pro-GRP, SCLC

\section{Introduction}

Lung cancer is one of the most common malignant tumors. In recent decades, its incidence has been increasing, especially among women, while its mortality rate has shown a slight reduction, mainly thanks to surgical and medical improvements (1). When approaching a newly detected lung cancer, treatments strictly depend on the histological type. In particular, an important step to be made is to distinguish between non-small cell lung cancer (NSCLC) and small cell lung cancer (SCLC). The management and the prognosis of

Received: July 26, 2017

Accepted: September 14, 2017

Published online: September 25, 2017

Corresponding author:

Dr. Stefano Cavalieri

Medical Oncology Department - National Cancer Institute of Milan

Fondazione IRCCS Istituto Nazionale Tumori

Via Venezian, 1

20133 Milan, Italy

stefano.cavalieri@istitutotumori.mi.it the former type depends mainly on histology (squamous cell cancer and adenocarcinoma being the most frequent types), clinical and pathological stage, the patient's comorbidities and performance status $(2,3)$. In contrast, SCLC has a poor prognosis and any treatments, whether chemotherapy or radiotherapy or both, have only a palliative intent (4).

Circulating biomarkers are available for lung cancer, but none of them are able to replace the role of histology in the definition of the pathology - indeed tumor biopsy is mandatory as a first step in lung cancer management. The elevation of some serum markers can be observed in both SCLC and NSCLC, while others are more associated with specific histopathological types. Neuron-specific enolase (NSE) is a valid marker for SCLC and its identification by immunohistochemical methods in tumor samples is an important step to confirm the diagnosis (5). Its accuracy increases with the coexpression of other neuroendocrine markers (chromogranin A and synaptophysin) and with the absence of epithelial and glandular antigens, such as cytokeratins for squamous cell carcinoma and thyroid transcription factor 1 (TTF-1) for adenocarcinoma (6). Circulating NSE can be elevated in cases of SCLC, but its specificity (Sp) and sensitivity $(\mathrm{Sn})$ are not sufficient to consider such a marker as pathognomonic for diagnosis. A weak predictive role and 
Pro-GRP in small cell lung cancer

no prognostic significance were reported for this marker when evaluated to assess response to chemotherapy (7).

High circulating levels of carcinoembryonic antigen (CEA) can be related to lung adenocarcinoma (8) and SCLC (9). However, this marker is more typical of colorectal cancer (10), although elevated CEA levels can be observed in many nonneoplastic conditions as well (11).

Another marker observed in lung cancer patients is CYFRA21-1, a peptidic fragment of cytokeratin-19. This peptide can be elevated in sera of NSCLC patients, but has a limited clinical utility in SCLC (12). Both CEA and CYFRA21-1 have been shown to have a predictive role in monitoring response to therapies for NSCLC (13). The identification of biomarkers having an analogous role for SCLC is still an unmet clinical need. Pro-gastrin-releasing peptide (pro-GRP) is an emerging circulating biomarker useful in SCLC diagnosis (14-16). It is the precursor of gastrin-releasing peptide (GRP), an active hormone involved in the physiological digestive process. GRP has shown mitogenic activity in vitro for SCLC, but not for squamous cell cancer or adenocarcinoma of the lung (17). GRP, the direct derivative of pro-GRP, might act as an autocrine growth factor for SCLC cells and GRP receptor mRNA is found in two fifths of cases of SCLC (18). The first identification of GRP in lung cancer specimens was in 1983. Its main limitation is its short half-life of approximately 2 minutes (19). Therefore laboratory assays have been developed in recent decades to identify its precursor pro-GRP, which is much more stable (20).

Many laboratory studies and patient series have been reported so far, but few data are available about the accuracy of pro-GRP in day-to-day clinical practice. The aim of this study was to evaluate single-center records of this validated marker in white patients. This paper discusses those results, with an overview of the literature.

\section{Methods}

\section{Patient selection}

This was a retrospective analysis of patients treated at our center for advanced lung cancer. Advanced disease was defined as the presence of unresectable or locally advanced or metastatic disease not amenable to locoregional treatments, in particular surgery and radiotherapy. Serum pro-GRP was measured in all patients with a newly diagnosed advanced lung cancer who were hospitalized in our Medical Oncology Unit from December 2014 to January 2017. CEA and NSE were measured in all patients as well.

\section{Assay description}

Serum pro-GRP, CEA and NSE were measured by an electrochemiluminescence immunoassay using a Roche C6000 automated analyzer (Roche Diagnostics $\mathrm{GmbH}$, Penzberg, Germany). For pro-GRP, the reference range was set as the interval between 0 and $77.8 \mathrm{pg} / \mathrm{mL}\left(77.8 \mathrm{pg} / \mathrm{mL}\right.$ was the $97.5^{\text {th }}$ percentile of serum pro-GRP concentration in 698 healthy white adults, as described in Roche studies No. RD001525 and RD000788 and as reported on the product sheet of the automated analyzer) (21). CEA and NSE were considered positive if their serum levels were higher than $5 \mathrm{ng} / \mathrm{mL}$ (9) and $16.3 \mathrm{ng} / \mathrm{mL}$ (22), respectively.

\section{Statistical analysis}

To calculate $\mathrm{Sn}, \mathrm{Sp}$, positive and negative predictive values (PPV and NPV, respectively), the following conditions were considered: SCLC with elevated marker as true positive (TP), NSCLC with normal marker as true negative (TN), SCLC with normal marker as false negative (FN) and NSCLC with elevated marker as false positive (FP). The same analysis was performed to discriminate limited (LD) from diffuse SCLC disease (DD). Supplementary Table I reports the definitions used for calculations (available online at www.biological-markers.com Definitions used to calculate sensitivity, specificity, positive and negative predictive values). The diagnostic odds ratio was calculated as a statistical tool combining both $\mathrm{Sn}$ and Sp in a single indicator (23). Receiver operating characteristic (ROC) curve analysis was completed to define threshold values for pro-GRP. The area under the ROC curve (AUC) was estimated, to quantify the overall accuracy of pro-GRP levels and their Sn and Sp.

Univariate analyses were performed to assess the role of liver metastases and renal impairment as potential confounders of serum pro-GRP levels. In all cases, categorical data were analyzed with chi-squared or Fisher's exact test and continuous variables with the Mann-Whitney $U$ test. Threshold for statistical significance was set at 0.05 . Statistical analyses were performed with GraphPad Prism ${ }^{\circledR}$, version 5.02.

\section{Results}

\section{Patient characteristics}

A total of 65 patients were analyzed: 37 patients with SCLC and 28 with locally advanced or metastatic NSCLC. All patients were Caucasian, three quarters of them were men, with a median age of 68 years. Patients' characteristics are summarized in Supplementary Table II (available online at www.biological-markers.com - Patient characteristics). In the majority of NSCLC patients (86\%) disease stage was IV according to the American Joint Committee on Cancer classification ( $8^{\text {th }}$ edition of the AJCC staging manual), while in the remaining 4 patients, tumor extension was locally advanced. ProGRP was measured in all patients. NSE values were available for 51 subjects (32 SCLC + 19 NSCLC), CEA for 43 (28 SCLC + 15 NSCLC).

\section{SCLC vs. NSCLC}

Median pro-GRP was $67 \mathrm{pg} / \mathrm{mL}$ (range 10-147,350) in the total study population, $919 \mathrm{pg} / \mathrm{mL}$ (range 22-147,350) in patients with SCLC and $32 \mathrm{pg} / \mathrm{mL}$ (range 10-119) in those affected by NSCLC $(p<0.0001)$. In SCLC patients, median NSE was 4.38 -fold higher than in NSCLC patients $(p=0.0005)$. Median CEA was $8.02 \mathrm{ng} / \mathrm{mL}$ (range 1.01-1,767) in SCLC and $9 \mathrm{ng} / \mathrm{mL}$ (range $0-241$ ) in NSCLC ( $p=0.98)$. Figure 1 summarizes the results on a logarithmic scale.

Considering local cutoff values for positivity $(77.8 \mathrm{pg} / \mathrm{mL})$, pro-GRP Sn, Sp and accuracy in SCLC were $86.49 \%, 96.43 \%$ and $90.77 \%$, respectively. Accuracy was $72.55 \%$ for NSE (Sn 

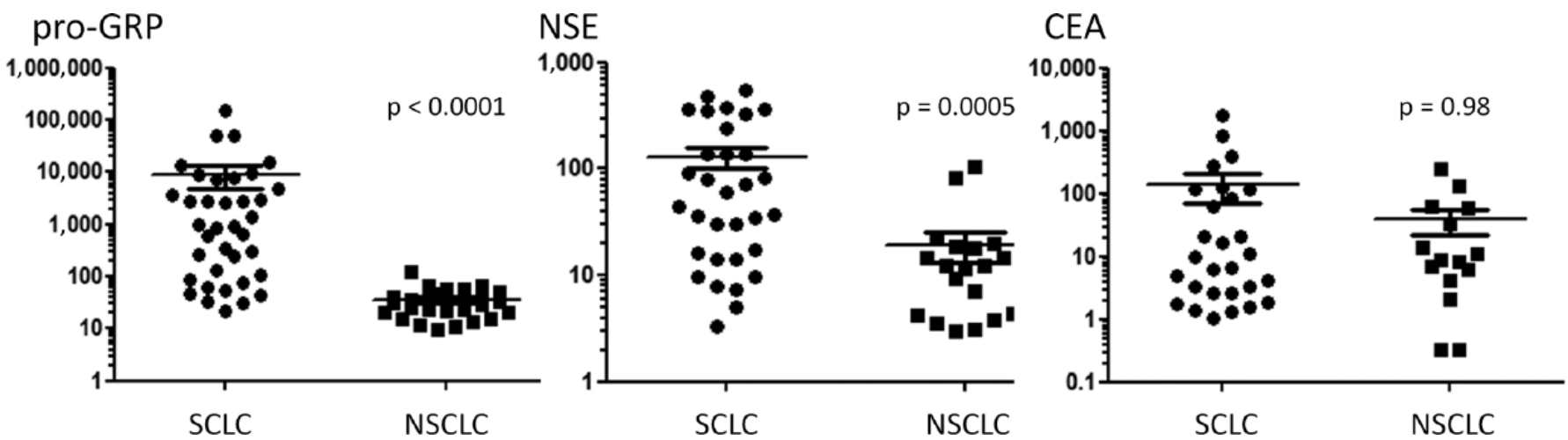

Fig. 1 - Serum marker values (small cell lung cancer [SCLC] vs. non-small cell lung cancer [NSCLC]). CEA = carcinoembryonic antigen; NSE = neuron-specific enolase; pro-GRP = pro-gastrin-releasing peptide.
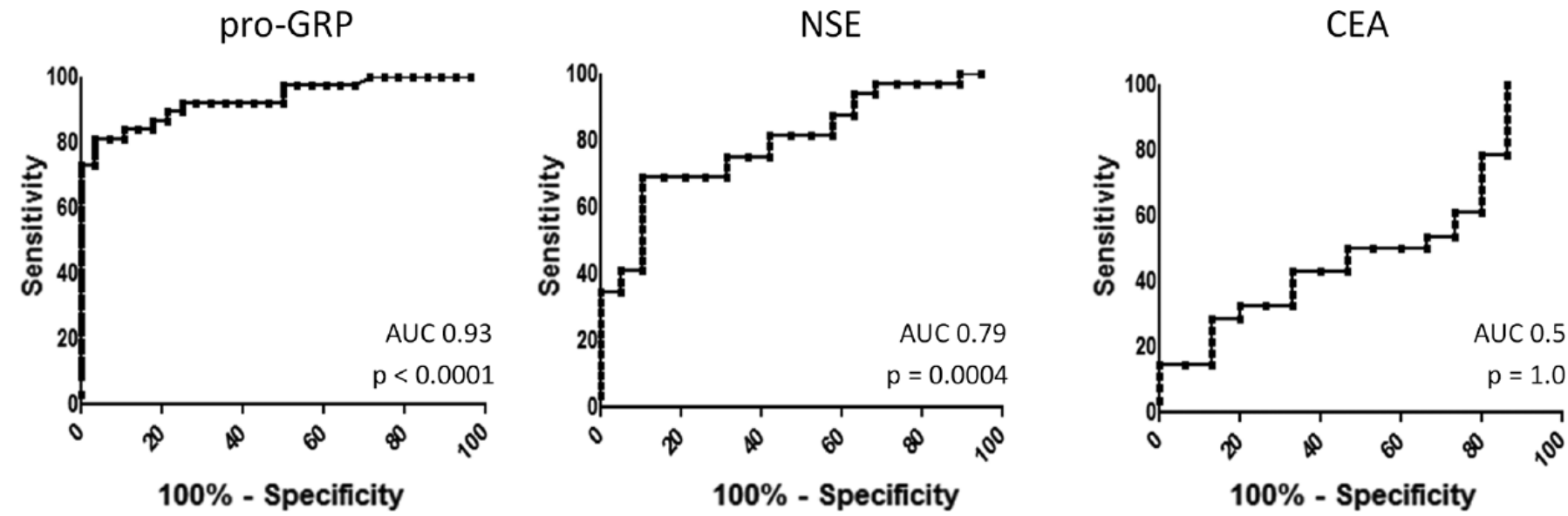

Fig. 2 - Receiver operator characteristic (ROC) curve analysis for markers small cell lung cancer (SCLC) vs. non-small cell lung cancer (NSCLC). $A U C=$ area under the curve; $C E A=$ carcinoembryonic antigen; NSE = neuron-specific enolase; pro-GRP = pro-gastrin-releasing peptide.

75\%, Sp 68.42\%), 46.51\% for CEA (Sn 57.14\%, Sp 26.67\%). These results are summarized in Supplementary Table III (available online at www.biological-markers.com - Pro-GRP sensitivity, specificity and accuracy in SCLC). On ROC curve analysis, the AUC was 0.93 (95\% confidence interval [95\% Cl], $0.87-0.99)$ for pro-GRP in SCLC $(p<0.0001)$. The serum value showing the highest likelihood ratio (22.7), thus potentially being a reliable threshold value, was $71.9 \mathrm{pg} / \mathrm{mL}$. At this marker concentration, Sn was $81 \%$ (95\% Cl, 64.8\%-92\%) and Sp 96.4\% (95\% Cl, 81.6\%-99.9\%). NSE and CEA AUCs were $0.79(95 \% \mathrm{Cl}, 0.67-0.91)$ and $0.5(95 \% \mathrm{Cl}, 0.31-0.68)$, respectively (Fig. 2). Five SCLC patients had normal pro-GRP values, thus they were considered as FNs. Four of them had elevated NSE (median NSE $13.9 \mathrm{ng} / \mathrm{mL}$, range 7.9-71.3) and two thirds (CEA not available for 2 cases) had elevated CEA (median CEA $6.07 \mathrm{ng} / \mathrm{ml}$, range 1.4-9.6). In particular, 2 patients had both positive markers, CEA and NSE were both negative in 1 patient and in the remaining 2 patients with elevated NSE, CEA results were not available.

Excluding patients with locoregional disease (8 with LD SCLC and 4 with NSCLC with unresectable locally advanced disease), the differences between pro-GRP values were statistically significant $(p<0.0001)$. In fact, median pro-GRP was
$2,659 \mathrm{pg} / \mathrm{mL}$ (range 31.6-147,350) in metastatic SCLC patients $(\mathrm{n}=29)$ and $34.85 \mathrm{pg} / \mathrm{mL}$ (range $9.5-119.2$ ) in NSCLC patients $(n=24)$ with metastatic disease.

\section{Diffuse vs. limited disease in SCLC}

Among SCLC patients, 29 suffered from DD and 8 from LD. Considering all of the limitations of a statistical analysis conducted over a small-size population, median pro-GRP was 46fold higher (Fig. 3) in the former group (DD 2,659 pg/ml, range 31.6-147,350; LD $57.85 \mathrm{pg} / \mathrm{mL}$, range 22-3702). This difference was statistically significant $(p=0.004)$. Median NSE and CEA values were higher in patients with metastatic disease than in those with locoregional tumors, but no statistically significant differences were observed ( $p=0.12$, for NSE; $p=0.3$, for CEA).

The same data were confirmed by ROC curve analysis as well. AUC was 0.83 (95\% Cl, 0.67-0.99) for pro-GRP in DD ( $p=$ $0.004)$. On ROC curve analysis, a threshold value of $329.3 \mathrm{pg} / \mathrm{mL}$ showed the highest likelihood ratio (6.07) in discriminating LD from DD. At this value, Sn was $75.8 \%$ (95\% Cl, 56.4\%-89.7\%) and $\mathrm{Sp} 87.5 \%(95 \% \mathrm{Cl}, 47.3 \%-99.6 \%)$. Using the validated threshold value of $77.8 \mathrm{pg} / \mathrm{mL}$ (see "Methods"), Sn and Sp for pro-GRP in discriminating DD from LD were $93.1 \%$ and $62.5 \%$, 

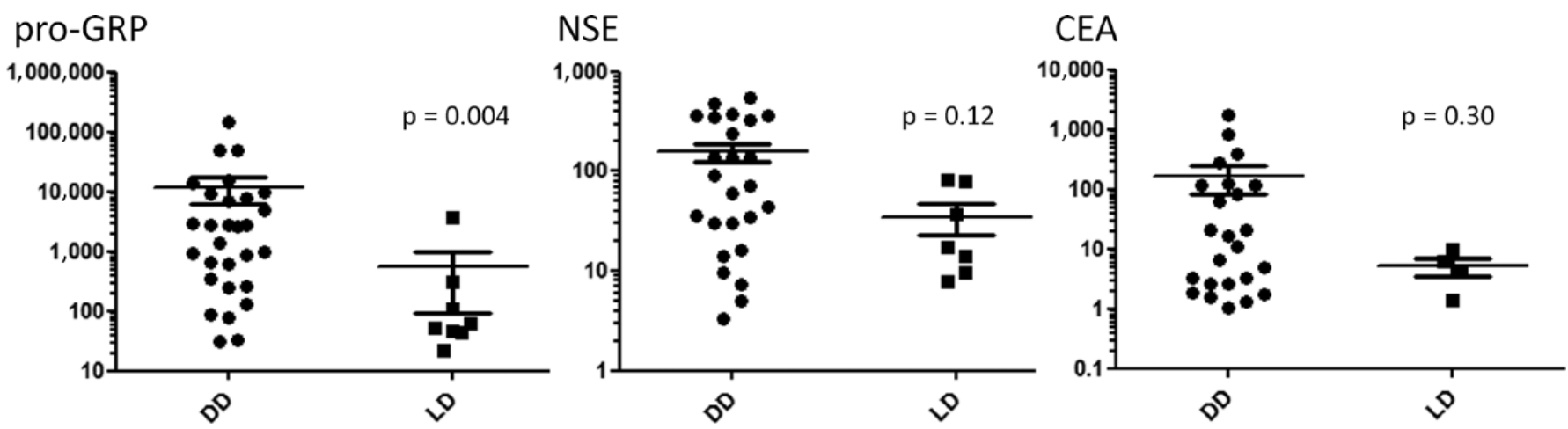

Fig. 3 - Serum marker values (diffuse [DD] vs. limited disease [LD] in small cell lung cancer [SCLC]). CEA = carcinoembryonic antigen; NSE = neuron-specific enolase; pro-GRP = pro-gastrin-releasing peptide.

respectively. These values were inferior for both NSE and CEA (Suppl Tab. IV, available online at www.biological-markers. com - Sensitivity and specificity for pro-GRP, NSE and CEA, in discriminating DD from LD).

\section{Serial marker measurements}

Serial measurements of serum pro-GRP levels were available for 19 patients (Fig. 4). The first data point is the baseline measurement, the following values were measured at each hospitalization for chemotherapy. Therefore, each point corresponds to a chemotherapy cycle. Figure $4 A, 4 B$ and $4 C$ reports the serial measurement of responders, patients with stable disease and non responding patients, respectively. In most cases an initial reduction was observed after the start of chemotherapy. With stable disease, no significant marker fluctuations were seen and in all patients disease progression was accompanied by pro-GRP elevation. Median reduction from baseline pro-GRP to first measurement after chemotherapy start was 0.24 (range -1.09 to 2.8 ). In 3 cases, a Log10 reduction was observed after systemic treatment and in 1 out of these 3 subjects a 664-fold reduction was observed (from 147,350 to $221.8 \mathrm{pg} / \mathrm{mL}$ ).

\section{Confounding factors}

Considering the total study population in a pooled manner, 19 patients had liver metastases (14 SCLC, 5 NSCLC). Renal function was reduced in 12 subjects (9 SCLC, 3 NSCLC). No significant roles of potentially confounding factors were observed. Pro-GRP was similar in both men and women $(p=0.34)$ and did not vary according to age $(p=$ 0.61 ). Median pro-GRP was 5.8-fold higher in patients with liver metastases than in those without hepatic disease, but the difference was statistically not significant $(p=0.32)$ and the AUC identified by ROC curve analysis was 0.59 . Patients with a reduced renal function (defined as an estimated glomerular filtration rate $[\mathrm{eGFR}]<90 \mathrm{~mL} / \mathrm{min}$ per body surface area [BSA], calculated with the Chronic Kidney Disease Epidemiology Collaboration [CKD-EPI] formula) had a slightly higher median pro-GRP value (1.35-fold higher), but also in this case, the difference was not statistically significant ( $p=0.45, A \cup C=0.58)$.

\section{Discussion}

Pro-GRP accuracy in SCLC was described for the first time in 1995, when this marker, measured with enzyme-linked immunosorbent assay, was reported to be higher in SCLC than in benign pneumopathies and in NSCLC (20).

The higher Sn and Sp of this marker in SCLC, in comparison with NSE, were confirmed by more recent studies as well (24). A better accuracy in the diagnosis of SCLC was reported for pro-GRP compared with NSE. Our data are in line with such results. Nonetheless, due to the scarcity of perspective data, to date the role of NSE cannot be superseded by pro-GRP.

In Bayesian statistics, we are used to thinking of a true negative as an event in which the study participant is a healthy control and the variable being studied is also negative. Even though the way we exploited to calculate sensitivity, specificity, PPV, NPV and also to discriminate between LD and DD is not usual, we considered only lung cancer patients in order to better adhere to a real life medical oncology clinical practice. Indeed, it is infrequent that such biomarkers could be used in an extra-oncologic setting.

The $\mathrm{Sn}$ of this marker was much higher in our population than that reported in the literature ( $86 \%$ vs. $47 \%$ ), while the Sp was comparable (25). Likely, patient selection bias could have influenced such results. Indeed, all patients in exam were affected by lung cancer and we considered as true negative only NSCLC patients with low marker values. On the contrary, to validate pro-GRP accuracy, literature reports analyzed as control population patients with benign lung diseases, such as sarcoidosis, chronic obstructive pulmonary disease and primary pulmonary fibrosis. In such a setting, to discriminate between SCLC and cancer-free patients, a lower cutoff level (34.2 pg/mL) was defined (26).

Another study showed higher threshold values for positivity $(50 \mathrm{pg} / \mathrm{mL})$, reporting that median serum values were $<80 \mathrm{pg} / \mathrm{mL}$ in benign lung diseases and $<120 \mathrm{pg} / \mathrm{mL}$ in patients with non lung and non neuroendocrine cancers (27). The cutoff value for pro-GRP positivity used in our laboratory and in this study was $77.8 \mathrm{pg} / \mathrm{mL}$. Considering the ROC curve analysis results, $71.9 \mathrm{pg} / \mathrm{mL}$ could be seen as a better threshold value to discriminate SCLC from NSCLC. The AUC and the diagnostic odds ratio revealed the robustness of pro-GRP in discriminating SCLC from NSCLC. Among the 3 tested markers, pro-GRP 
A

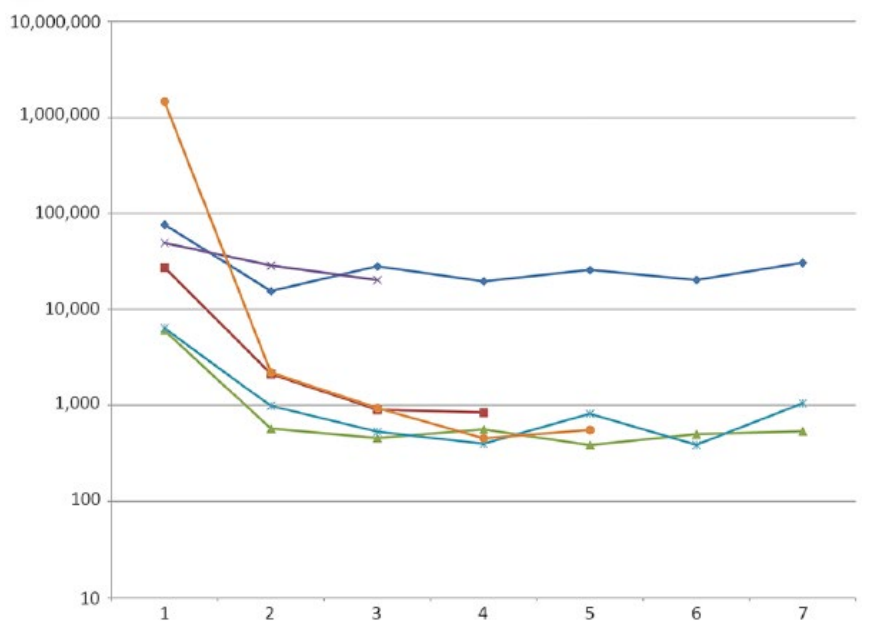

B

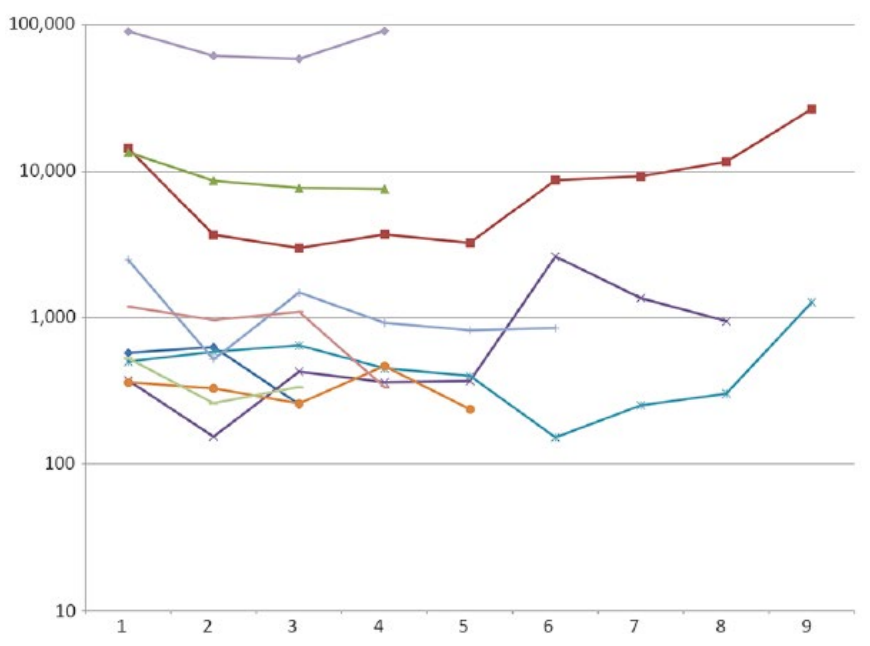

C

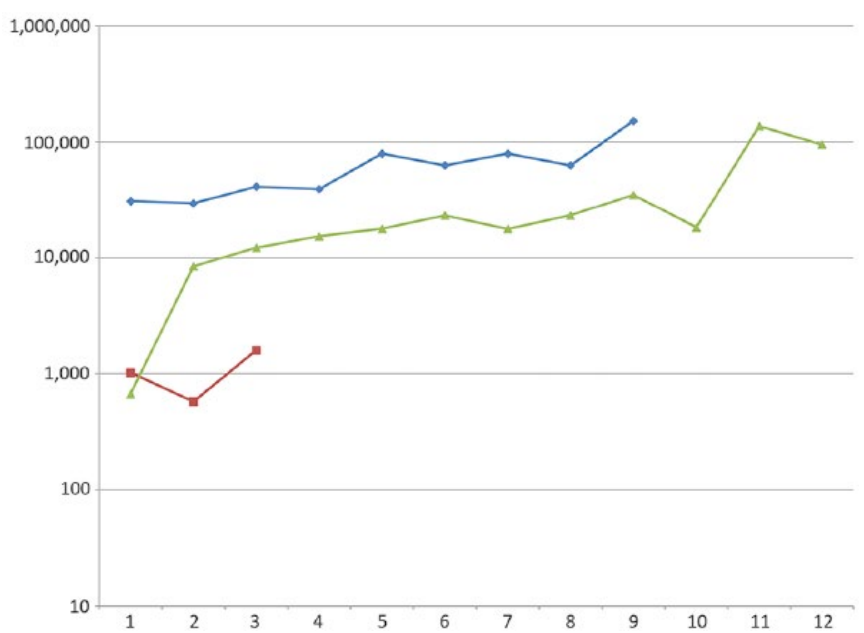

Fig. 4 - Serial measurements of serum pro-GRP in 19 small cell lung cancer (SCLC) patients (Log10 scales): (A) patients with response to therapy $(n=6) ;(B)$ patients with stable disease $(n=10)$; and (C) patients with disease progression $(n=3)$. had the highest AUC and diagnostic odds ratio, followed by NSE and CEA.

Among the 5 SCLC patients with normal pro-GRP (FN), 3 were affected by LD and 2 had oligometastatic disease. Due to the NSE positivity in of 4 of these 5 cases, NSE should be used as complementary marker, as already suggested by other authors, while skepticism about the utility of CEA remains when both NSE and pro-GRP evaluations are performed (28).

A limitation of our study was the low statistical power due to the small sample size. Nonetheless, we used statistical methods to find any differences between LD and DD to have just an outline of what is intuitively observed in dayto-day clinical practice - that is, an even apparent correlation between tumor burden and marker levels. The results of our analysis are, of course, preliminary, because a correct statistical analysis could not be performed using our sample size.

The only NSCLC patient with a high pro-GRP level (119.2 pg/mL) was affected by a poorly differentiated lung adenocarcinoma with a high burden of metastatic disease (FP). In this patient, CEA was higher than the upper reference limit $(8.59 \mathrm{ng} / \mathrm{mL})$, while NSE was normal (3.1 ng/mL).

In the setting of SCLC, no role for pro-GRP in predicting distant metastasis has been described so far, therefore the measurement of such a marker should avoided in this setting at baseline (29). On the other hand, some roles in predicting neurological events and survival were found for pro-GRP in patients undergoing prophylactic cranial irradiation (30).

Our data suggest that a serum pro-GRP higher than $329.3 \mathrm{pg} / \mathrm{mL}$ might have a satisfactory Sp with a modest Sn in helping to differentiate DD from LD SCLC. These data can be interpreted as showing that vast tumor burdens could be related to higher pro-GRP production. Therefore, the accuracy of such a marker is comparable to that shown in reports in the scientific literature (31).

Within the limitations of a retrospective analysis, we observed that pro-GRP levels were related to disease natural history. Indeed, we observed marker increases in case of disease progression, reductions in case of response to treatments and fluctuations in case of stable disease. In an Asian case series considering 82 patients with unselected lung cancer (both SCLC and NSCLC), pro-GRP was reduced after chemotherapy start, but the biomarker's accuracy was not validated (32). On the other hand, both serum pro-GRP and NSE failed to be established as markers for detecting relapses before the onset of overt clinical or radiographic findings (33). Perspective studies with white patients confirmed these results (34). In another case series, the most reliable circulating marker in monitoring response to treatments was pro-GRP, because NSE, CYFRA 21-1 and lactate dehydrogenase levels fluctuate during systemic therapies (35).

A recent study described the role of both pro-GRP and NSE not only in monitoring treatment response, but also in predicting patients' survival (36). An association with survival was reported with univariate analysis of limited SCLC (37). A retrospective analysis showed that pro-GRP revealed a higher correlation to tumor shrinkage and to prognosis than NSE (38). It is possible that the correlation between this marker and survival can be due to underlying massively extended disease and/or high disease burden. 
The role of chronic kidney failure in altering serum biomarkers has been reported, notably for CYFRA21-1 and pro-GRP (39). Nonetheless, within the limitations of a low statistical power, our data showed that neither the presence of liver metastases nor a concomitant renal impairment influenced pro-GRP levels in SCLC patients. An eGFR of $60 \mathrm{~mL} / \mathrm{min}$ per BSA is the usual cutoff value used to define an impaired renal function. We deliberately chose to set it at $90 \mathrm{~mL} / \mathrm{min}$ per BSA, to test differences in pro-GRP values between people with a normal blood creatinine and patients with any modest renal damage. It is likely that higher pro-GRP values observed in patients with liver metastases, even though not statistically significant, could be related to higher systemic disease burden rather than a reduced pro-GRP altered metabolism.

As reported before, pro-GRP products, notably GRP, are able to induce cell progression in SCLC. Recent studies revealed that GRP down-regulation is able to reduce SCLC growth and to increase tumor cell apoptosis. Therefore, since GRP inhibition could constitute a theoretical target for cancer treatments, further study evaluating the role of pro-GRP should be conducted (40).

\section{Conclusion}

Our data and review of the literature showed that pro-GRP can be considered as an affordable and accurate biomarker in the diagnosis of SCLC and a useful tool to discriminate SCLC from NSCLC. Due to our small sample size, no inferences can be made in discerning DD from LD. The Sn and Sp of pro-GRP are higher than those for NSE and CEA. Its utility in monitoring treatment response is confirmed by literature data. An interesting point to be clarified in the future would be the investigation of this biomarker in monitoring the response to new therapeutic agents.

\section{Disclosures}

Financial support: No funding was received by the authors for this work.

Conflict of interest: There are no conflicts of interest to declare regarding this study.

\section{References}

1. Ferlay J, Soerjomataram I, Dikshit R, et al. Cancer incidence and mortality worldwide: sources, methods and major patterns in GLOBOCAN 2012. Int J Cancer. 2015;136(5):E359-E386.

2. Vansteenkiste J, De Ruysscher D, Eberhardt WE, et al. ESMO Guidelines Working Group. Early and locally advanced nonsmall-cell lung cancer (NSCLC): ESMO clinical practice guidelines for diagnosis, treatment and follow-up. Ann Oncol. 2013;24(Suppl 6):vi89-vi98.

3. Novello S, Barlesi F, Califano R, et al. ESMO Guidelines Committee. Metastatic non-small-cell lung cancer: ESMO clinical practice guidelines for diagnosis, treatment and follow-up. Ann Oncol. 2016;27(Suppl 5):v1-v27.

4. Früh $M$, De Ruysscher D, Popat $S$, Crinò L, Peters S, Felip E; ESMO Guidelines Working Group. Small-cell lung cancer (SCLC): ESMO clinical practice guidelines for diagnosis, treatment and follow-up. Ann Oncol. 2013;24(Suppl 6):vi99-vi105.

5. OShea P, Cassidy M, Freaney R, McCarthy P, Fennelly J. Serum neuron-specific enolase and immunohistochemical markers of neuroendocrine differentiation in lung cancer. Ir J Med Sci. 1995;164(1):31-36.

6. Au NH, Cheang M, Huntsman DG, et al. Evaluation of immunohistochemical markers in non-small cell lung cancer by unsupervised hierarchical clustering analysis: a tissue microarray study of 284 cases and 18 markers. J Pathol. 2004;204(1):101-109.

7. Tiseo M, Ardizzoni A, Cafferata MA, et al. Predictive and prognostic significance of neuron-specific enolase (NSE) in nonsmall cell lung cancer. Anticancer Res. 2008;28(1B):507-513.

8. Grunnet M, Sorensen JB. Carcinoembryonic antigen (CEA) as tumor marker in lung cancer. Lung Cancer. 2012;76(2):138-143.

9. Lokich JJ. Plasma CEA levels in small cell lung cancer: correlation with stage, distribution of metastases and survival. Cancer. 1982;50(10):2154-2156.

10. Tan E, Gouvas N, Nicholls RJ, Ziprin P, Xynos E, Tekkis PP. Diagnostic precision of carcinoembryonic antigen in the detection of recurrence of colorectal cancer. Surg Oncol. 2009;18(1): 15-24.

11. Kang HY, Choe EK, Park KJ, Lee Y. Factors requiring adjustment in the interpretation of serum carcinoembryonic antigen: a cross-sectional study of 18,131 healthy nonsmokers. Gastroenterol Res Pract. 2017;2017:9858931.

12. Zhang L, Liu D, Li L, et al. The important role of circulating CY FRA211 in metastasis diagnosis and prognostic value compared with carcinoembryonic antigen and neuron-specific enolase in lung cancer patients. BMC Cancer. 2017;17(1):96.

13. Holdenrieder $S$, Wehnl B, Hettwer $K$, Simon $K$, Uhlig $S$, Dayyani F. Carcinoembryonic antigen and cytokeratin-19 fragments for assessment of therapy response in non-small cell lung cancer: a systematic review and meta-analysis. $\mathrm{Br} J$ Cancer. 2017;116(8):1037-1045.

14. Miyake $Y$, Kodama T, Yamaguchi K. Pro-gastrin-releasing peptide(3198) is a specific tumor marker in patients with small cell lung carcinoma. Cancer Res. 1994;54(8):2136-2140.

15. Takada M, Kusunoki $Y$, Masuda N, et al. Pro-gastrin-releasing peptide (3198) as a tumour marker of small-cell lung cancer: comparative evaluation with neuron-specific enolase. Br J Cancer. 1996;73(10):1227-1232.

16. Shibayama $\mathrm{T}$, Ueoka $\mathrm{H}, \mathrm{Nishii} \mathrm{K}$, et al. Complementary roles of pro-gastrin-releasing peptide (ProGRP) and neuron specific enolase (NSE) in diagnosis and prognosis of small-cell lung cancer (SCLC). Lung Cancer. 2001;32(1):61-69.

17. Weber S, Zuckerman JE, Bostwick DG, Bensch KG, Sikic BI, Raffin TA. Gastrin releasing peptide is a selective mitogen for small cell lung carcinoma in vitro. J Clin Invest. 1985;75(1):306-309.

18. Uchida K, Kojima A, Morokawa N, et al. Expression of progastrin-releasing peptide and gastrin-releasing peptide receptor mRNA transcripts in tumor cells of patients with small cell lung cancer. J Cancer Res Clin Oncol. 2002;128(12):633-640.

19. Yamaguchi K, Abe K, Kameya T, et al. Production and molecular size heterogeneity of immunoreactive gastrin-releasing peptide in fetal and adult lungs and primary lung tumors. Cancer Res. 1983;43(8):3932-3939.

20. Yamaguchi K, Aoyagi K, Urakami K, et al. Enzyme-linked immunosorbent assay of pro-gastrin-releasing peptide for small cell lung cancer patients in comparison with neuron-specific enolase measurement. Jpn J Cancer Res. 1995;86(7):698-705.

21. Elecsys ${ }^{\circledR}$ ProGRP Roche product sheet: electro-chemiluminescence immunoassay (ECLIA) for the quantitative determination of human progastrinreleasing peptide (ProGRP) in serum and plasma [package insert]. Penzberg, Germany: Roche. http:// www.cobas.com/content/dam/cobas_com/pdf/product/ Elecsys\%20ProGRP\%20assay/ProGRP\%20fact\%20sheet.pdf Accessed September 07, 2017.

22. Suh KJ, Keam B, Kim M, et al. Serum neuron-specific enolase levels predict the efficacy of first-line epidermal growth factor 
receptor (EGFR) tyrosine kinase inhibitors in patients with nonsmall cell lung cancer harboring EGFR mutations. Clin Lung Cancer. 2016;17(4):245-252.

23. Glas AS, Lijmer JG, Prins MH, Bonsel GJ, Bossuyt PM. The diagnostic odds ratio: a single indicator of test performance. J Clin Epidemiol. 2003;56(11):1129-1135.

24. Schneider J, Philipp M, Velcovsky HG, Morr H, Katz N. Progastrin-releasing peptide (ProGRP), neuron specific enolase (NSE), carcinoembryonic antigen (CEA) and cytokeratin 19-fragments (CYFRA 211) in patients with lung cancer in comparison to other lung diseases. Anticancer Res. 2003;23(2A):885-893.

25. Stieber $P$, Dienemann $H$, Schalhorn A, et al. Pro-gastrin-releasing peptide (ProGRP), a useful marker in small cell lung carcinomas. Anticancer Res. 1999;19(4A):2673-2678.

26. Oremek GM, Sapoutzis N. Pro-gastrin-releasing peptide (ProGRP), a tumor marker for small cell lung cancer. Anticancer Res. 2003;23(2A):895-898.

27. Molina R, Filella $X$, Augé JM. ProGRP: a new biomarker for small cell lung cancer. Clin Biochem. 2004;37(7):505-511.

28. Wang H, Qian J. Serum pro-gastrin-releasing peptide in diagnosis of small cell lung cancer: a meta-analysis. J Cancer Res Ther. 2016;12(8)(Suppl):C260-C263.

29. Li BT, Lou E, Hsu M, et al. Serum biomarkers associated with clinical outcomes fail to predict brain metastases in patients with stage IV non-small cell lung cancers. PLoS ONE. 2016;11(1):e0146063.

30. Yonemori K, Sumi M, Fujimoto $\mathrm{N}$, et al. Pro-gastrin-releasing peptide as a factor predicting the incidence of brain metastasis in patients with small cell lung carcinoma with limited disease receiving prophylactic cranial irradiation. Cancer. 2005; 104(4):811-816.

31. Wang L, Wang D, Zheng G, et al. Clinical evaluation and therapeutic monitoring value of serum tumor markers in lung cancer. Int J Biol Markers. 2016;31(1):e80-e87.
32. Wang J, Shi G, Zhang S, et al. [Clinical value of serum TPS, CEA Pro-GRP and CYFRA211 in patients with lung cancer] [article in Chinese]. Zhongguo Fei Ai Za Zhi. 2010;13(5):500-505.

33. Hirose T, Okuda K, Yamaoka T, et al. Are levels of pro-gastrin-releasing peptide or neuron-specific enolase at relapse prognostic factors after relapse in patients with small-cell lung cancer? Lung Cancer. 2011;71(2):224-228.

34. Schneider J, Philipp M, Salewski L, Velcovsky HG. Pro-gastrinreleasing peptide (ProGRP) and neuron specific enolase (NSE) in therapy control of patients with small-cell lung cancer. Clin Lab. 2003;49(1-2):35-42.

35. Wójcik E, Kulpa JK, Sas-Korczyńska B, Korzeniowski S, Jakubowicz J. ProGRP and NSE in therapy monitoring in patients with small cell lung cancer. Anticancer Res. 2008;28(5B):3027-3033.

36. Huang $Z, X u D$, Zhang F, Ying $Y$, Song L. Pro-gastrin-releasing peptide and neuron-specific enolase: useful predictors of response to chemotherapy and survival in patients with small cell lung cancer. Clin Transl Oncol. 2016;18(10):1019-1025.

37. Nisman B, Biran H, Ramu N, Heching N, Barak V, Peretz T. The diagnostic and prognostic value of ProGRP in lung cancer. Anticancer Res. 2009;29(11):4827-4832.

38. Ono A, Naito T, Ito I, et al. Correlations between serial progastrin-releasing peptide and neuron-specific enolase levels and the radiological response to treatment and survival of patients with small-cell lung cancer. Lung Cancer. 2012;76(3): 439-444.

39. Nakahama H, Tanaka $Y$, Fujita $Y$, Fujii M, Sugita M. CYFRA 211 and ProGRP, tumor markers of lung cancer, are elevated in chronic renal failure patients. Respirology. 1998;3(3): 207-210.

40. Gong Z, Lu R, Xie S, et al. Overexpression of pro-gastrin releasing peptide promotes the cell proliferation and progression in small cell lung cancer. Biochem Biophys Res Commun. 2016;479(2):312-318. 